\title{
Sexual intercourse and risk of ischaemic stroke and coronary heart disease: the Caerphilly study
}

\section{S Ebrahim, M May, Y Ben Shlomo, P McCarron, S Frankel, J Yarnell, G Davey Smith}

J Epidemiol Community Health 2002;56:99-102

See end of article for authors' affiliations

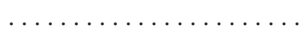

Correspondence to: Professor S Ebrahim, Department of Social Medicine, University of Bristol, Canynge Hall, Whiteladies Road, Bristol BS8 2PR, UK ;

shah.ebrahim@bristol.ac.uk

Accepted for publication 8 June 2001

\begin{abstract}
Objective: To examine the relation between frequency of sexual intercourse and risk of ischaemic stroke and coronary heart disease.

Design: Cohort study with 20 year follow up.

Setting: The town of Caerphilly, South Wales and five adjacent villages.

Subjects: 914 men aged 45-59 years at time of recruitment in 1979 to 1983.

Main outcome measures: Ischaemic stroke and coronary heart disease, all first events and fatal events.

Results: Of the 914 men studied, 197 (21.5\%) reported sexual intercourse less often than once a month, $231(25.3 \%)$ reported sexual intercourse twice or more a week, and the remaining 486 $(53.2 \%)$ men fell into the intermediate category. Frequency of sexual intercourse was not associated with all first ischaemic stroke events: age adjusted odds ratios $(95 \% \mathrm{Cl})$ for intermediate and low frequency of sexual intercourse of $0.61(0.32$ to 1.16$)$ and $0.71(0.34$ to 1.49$)$ respectively compared with the reference category of high frequency. A graded relation with fatal coronary heart disease events was observed in events recorded up to 10 years. The age adjusted relative risk $(95 \% \mathrm{CI})$ of fatal coronary heart disease contrasting low frequency of sexual intercourse (that is, less than monthly) with the highest group (at least twice a week) was 2.80 (1.13 to 6.96, test for trend, $p=0.04$ ) which was not attenuated by adjustment for a wide range of potential confounders. Longer follow up to 20 years showed attenuation of this risk with odds of 1.69 (95\% Cl 0.90 to 3.20), contrasting low frequency of sexual intercourse with the highest group.

Conclusions: The differential relation between frequency of sexual intercourse, stroke and coronary heart disease suggests that confounding is an unlikely explanation for the observed association with fatal coronary heart disease events. Middle aged men should be heartened to know that frequent sexual intercourse is not likely to result in a substantial increase in risk of strokes, and that some protection from fatal coronary events may be an added bonus.
\end{abstract}

A recent Japanese series of 42 patients suffering haemorrhagic stroke reported that in 23 cases prior extramarital sex was a suspected causal factor ${ }^{1}$ and excited considerable media attention despite its methodological weaknesses. The high levels of recent and past media interest in reported relations between sexual intercourse and cardiovascular disease do not reflect a merely prurient interest (see for example, Daily Express and Associated Press stories. ${ }^{2}{ }^{3}$ ) Rather, given the very meagre scientific literature available, media stories provide almost the only source of information to support or refute lay epidemiology.

As strokes occur quite commonly at night and in the early morning, coincidence with sexual activity may result in survivors and their partners assuming a causal association. None of the conventional risk factors vary between patients suffering stroke at night or in the morning compared with other times. ${ }^{45}$ An adverse relation with sexual activity remains a possibility, although a previous report demonstrated a halving of mortality among men reporting sexual intercourse frequency of more than twice a week; this relation was stronger for coronary heart disease mortality than for other causes of death, but non-fatal events and strokes were not considered. ${ }^{6}$ We hypothesised that men experiencing more sexual intercourse would be at greater risk of strokes and therefore sought to examine the relation between frequency of sexual intercourse and stroke incidence.

\section{METHODS}

Full details of the Caerphilly study are available elsewhere. ${ }^{78}$ Briefly, in Caerphilly a former mining town in South Wales, electoral rolls for the defined area were used to identify a random sample of men, each of whom was contacted and asked his age. Those aged $45-59$ years were invited to take part in the study. Of 2818 men identified 2513 (89\%) participated in the baseline survey-Phase I-in 1979-83. At recruitment a clinic assessment including a standard medical history, together with a wide range of variables including weight, height, blood pressure, and a 12 lead ECG. Married men were asked about frequency of sexual intercourse by a medical interviewer at Phase I. Responses were classified into categories ranging from never to daily and these were reduced to three categories: less than monthly, twice a week or more, and an intermediate category. Recall of sexual intercourse has been shown to be a reliable measure of male sexual activity, correlating with plasma testosterone and other gonadotrophins, and showing an age related decline in a study of 220 men aged 41 to 93 years. ${ }^{9}$ The question was asked only in the first period of the survey and was abandoned because of concerns about affecting the overall response rate. Responses were provided by 914 men out of 1222 men interviewed before the question was withdrawn, the large majority (98\%) of whom were married.

\section{Identification of ischaemic stroke}

Over the ensuing 20 years at follow up examinations held every four to five years, men were asked about stroke and transient ischaemic attacks (TIAs). Initially these were simply included in a check list of possible conditions, but in later follow ups detailed questions about symptoms that might be attributable to stroke and TIA events were asked. 
Table 1 Risk factors by frequency of sexual intercourse

\begin{tabular}{|c|c|c|c|}
\hline \multirow[b]{2}{*}{ Risk factor } & \multicolumn{3}{|c|}{ Frequency of sexual intercourse } \\
\hline & High $(n=231)$ & Intermediate $(n=486)$ & Low $(n=197)$ \\
\hline Systolic blood pressure & $142.5(18.7)$ & $142.1(19.1)$ & $142.4(18.8)$ \\
\hline $\mathrm{FEV}_{1} /$ height $^{2}$ & $1.0(0.2)$ & $1.01(0.2)$ & $0.9(0.2)$ \\
\hline Blood cholesterol & $5.7(1.1)$ & $5.7(1.2)$ & $5.6(1.2)$ \\
\hline Fibrinogen & $3.7(0.9)$ & $3.7(0.8)$ & $3.9(0.9)$ \\
\hline Current smoking (\%) & $55.4(128)$ & $52.5(255)$ & $56.8\left(\begin{array}{lll}1 & 12\end{array}\right)$ \\
\hline Social class $\%$ manual ${ }^{*}$ & $56.7(131 / 229)$ & $56.2(273 / 479)$ & $67.0(132 / 188)$ \\
\hline Paternal social class manual $†$ & $78.4(181 / 212)$ & $74.9(364 / 438)$ & $81.7(161 / 181)$ \\
\hline Self reported hypertension (\%) & $15.6(36)$ & $13.6(66)$ & $23.9(47)$ \\
\hline ECG defined ischaemia (\%) & $2.2(5)$ & $3.1(15)$ & $5.6(11)$ \\
\hline Angina $(\%)$ & $5.2(12)$ & $7.4(36)$ & $8.1(16)$ \\
\hline
\end{tabular}

Information was sought on all possible stroke events including those that occurred before the beginning of the study. In addition, routine hospital activity data and relevant clinical and pathological reports were abstracted from hospital notes for all men suspected of suffering a stroke. General practitioner records were inspected for events that had not led to hospital admission. Radiology records of CT scans were searched on every subject known to have been admitted to hospital with a cerebral event. Postmortem records were also reviewed for possible stroke events. Summary information on each possible stroke was assessed by three independent doctors who rated each event as: not a stroke, ischaemic, haemorrhagic, TIA, not known. Further details of the methods used have been reported. ${ }^{8}$ Deaths from stroke comprised all those coded as ICD 430-8 (9th revision of the International Classification of Diseases) and were obtained from the NHS central registry.

\section{Identification of coronary heart disease events}

Incident coronary heart disease (new cases arising during follow up) were ascertained from death certificates, review of hospital notes, and electrocardiographic changes, using the same conventions as in previous prospective analyses of this cohort. ${ }^{10}$ Non-fatal coronary heart disease comprised clinical myocardial infarction (hospitalised episodes meeting WHO criteria of combinations of serial electrocardiographic changes, increased concentrations of cardiac enzymes, and acute symptoms); and development of new Q or QS waves (Minnesota codes $1-1-1$ to $1-2-5$, or $1-2-7$ ). Deaths were classified as attributable to coronary heart disease (ICD-9 codes 410-414) from certificates provided by NHS central registry. Stroke and coronary heart disease events were assessed without knowledge of the man's frequency of sexual intercourse.

\section{Statistical methods}

Logistic regression was used to explore the relation between frequency of sexual intercourse and stroke adjusting for age and a wide range of risk factors for stroke (systolic blood pressure, current smoking, FEVl/height ${ }^{2}$, blood cholesterol, fibrinogen, social class, paternal social class, self reported hypertension, ECG defined ischaemia, angina). Similar analyses were conducted for fatal and non-fatal coronary heart disease.

\section{RESULTS}

Data regarding frequency of sexual intercourse were available on 914 men (75\% response rate). Of these, 197 (21.5\%) reported sexual intercourse less often than once a month, 231 $(25.3 \%)$ reported sexual intercourse twice or more a week, and the remaining 486 (53.2\%) men fell into the intermediate category. Risk factor distributions by frequency of sexual intercourse are shown in table 1. Manual social class, self reported hypertension, ECG defined ischaemic, and angina showed an increasing trend from high to low frequency of sexual intercourse.

Over the 20 years of follow up, 65 of these 914 men suffered a first ischaemic stroke, or which 26 events were fatal. Table 2 shows the relation between frequency of sexual intercourse and all first and fatal ischaemic stroke events. Although the odds of stroke were slightly lower among those reporting the lowest frequency of sexual intercourse, no clear pattern of increased risk was seen. The age adjusted odds ratios for all first ischaemic stroke of intermediate and low frequency of sexual intercourse were 0.61 (95\% CI 0.32 to 1.16 ) and 0.71 (95\% CI 0.34 to 1.49 ). Further adjustment for a wide range of factors did not materially change these relations, although confidence intervals were wide. Among those men suffering fatal strokes, the odds of stroke were lower in those reporting intermediate and low levels of sexual intercourse. Stroke was more common in those men who did not respond to the question on sexual activity (fully adjusted OR 1.49 (0.84 to 2.65).

As treatment with antihypertensive drugs may cause impotence, we examined the relations in men taking and not taking these drugs. In men taking antihypertensives, stroke was

Table 2 Relation between frequency of sexual intercourse and ischaemic stroke

\begin{tabular}{|c|c|c|c|c|}
\hline Frequency of sexual intercourse & Number (\%) events & Age adjusted odds ratio $(95 \% \mathrm{Cl})$ & Fully adjusted* odds ratio & $\mathrm{p}$ value for trend \\
\hline \multicolumn{5}{|l|}{ All ischaemic stroke } \\
\hline High & $17(7.4 \%)$ & 1 & 1 & 0.35 \\
\hline Intermediate & $30(6.2 \%)$ & $0.61(0.32$ to 1.16$)$ & $0.64(0.33$ to 1.22$)$ & \\
\hline Low & $18(9.1 \%)$ & $0.71(0.34$ to 1.49$)$ & $0.68(0.32$ to 1.45$)$ & \\
\hline \multicolumn{5}{|l|}{ Fatal ischaemic stroke } \\
\hline High & $8(3.5 \%)$ & 1 & 1 & 0.10 \\
\hline Intermediate & $12(2.5 \%)$ & $0.49(0.19$ to 1.26$)$ & $0.46(0.17$ to 1.23$)$ & \\
\hline Low & $6(3.1 \%)$ & $0.44(0.14$ to 1.40$)$ & $0.35(0.10$ to 1.14$)$ & \\
\hline
\end{tabular}

*Adjusted for systolic blood pressure, current smoking, $\mathrm{FEV}, /$ height ${ }^{2}$, blood cholesterol, fibrinogen, social class, paternal social class, self reported hypertension, ECG defined ischaemia, angina. 
Table 3 Relation between frequency of sexual intercourse and CHD risk

\begin{tabular}{llll}
\hline Frequency of sexual intercourse & Number (\%) events & Age adjusted odds ratio $(95 \% \mathrm{Cl})$ & Fully adjusted odds ratio* \\
\hline All CHD & & & 1 \\
High value for trend & $37(16.0 \%)$ & 1 & $0.95(0.60$ to 1.50$)$ \\
Intermediate & $80(16.5 \%)$ & $0.92(0.59$ to 1.42$)$ & $1.40(0.82$ to 2.39$)$ \\
Low & $52(26.4 \%)$ & $1.47(0.89$ to 2.44$)$ & 1 \\
Fatal CHD & $19(8.2 \%)$ & 1 & $1.30(0.72$ to 2.35$)$ \\
High & $53(10.9 \%)$ & $1.19(0.68$ to 2.09$)$ & $1.53(0.77$ to 3.01$)$ \\
Intermediate & $33(16.8 \%)$ & $1.69(0.90$ to 3.20$)$ & 0.24 \\
Low & & & \\
\hline
\end{tabular}

*Adjusted for systolic blood pressure, current smoking, $\mathrm{FEV}_{1} /$ height ${ }^{2}$, blood cholesterol, fibrinogen, social class, paternal social class, self reported

hypertension, ECG defined ischaemia, angina.

somewhat less common in those with a low frequency of sexual intercourse compared with those reporting high or intermediate frequency (age adjusted OR 0.82 , 95\% CI 0.25 to 2.67). In those not taking antihypertensives and reporting a low frequency of sexual intercourse, stroke was slightly more common (age adjusted OR $1.23,95 \%$ CI 0.60 to 2.53). But in both groups, confidence intervals were wide and included no relation.

We also examined the relation between frequency of sexual intercourse and fatal and all first coronary heart disease events occurring up to 20 years (see table 3 ). Death was more common in those reporting an intermediate or low frequency of sexual intercourse (intermediate frequency age adjusted OR $-1.19,95 \%$ CI 0.68 to 2.09 and low frequency $-1.69,95 \%$ CI 0.90 to 3.20 ), showing a graded trend. All first coronary heart disease events showed a similar trend (intermediate frequency age adjusted OR 0.92 95\% CI 0.59 to 1.42, low frequency $1.47,95 \%$ CI 0.89 to 2.44 ). At 10 years of follow up, fatal CHD events were more than twice as common in those reporting an intermediate or low frequency of sexual intercourse (intermediate frequency age adjusted OR -2.07 , $95 \%$ CI 0.89 to 4.80 and low frequency $-2.80,95 \%$ CI 1.12 to $6.96)$, showing a stronger effect than after 20 years follow up.

\section{DISCUSSION}

This is the first study, to our knowledge, to examine the relation between frequency of sexual intercourse and risk of ischaemic stroke. The absence of any robust relation is reassuring for the public who might reasonably believe that sexual activity can cause strokes, although it must be acknowledged that the number of stroke events studied was small and confidence intervals were wide. Prospective studies provide the best means of examining causal hypotheses of this nature as evaluation of frequency of sexual intercourse was made before the onset of disease and fatal and non-fatal events ascertained over a long period of time. If we were to use information about sexual intercourse closer to the time of event (which we do not have), it is much more likely that reverse causality would operate-men would reduce their sexual activity because of cardiovascular disease. The attenuation of the relation with coronary heart disease mortality suggests that baseline levels of sexual intercourse probably declined over time.

A recent report from the US Physicians' Health Study ${ }^{11}$ concerning the relation between 23 sudden deaths occurring during or within 30 minutes of episodes of vigorous exertion found that sudden death was increased by 16.9-fold by such exertion. While specific types of activity were not recorded, this risk attenuated to 4.6 -fold between midnight and 6 am, but was consistently raised at all other times of the day. While these relative risks are high, and might be relevant to activity achieved during sexual intercourse, the absolute risks of sudden death are extremely small and represent one sudden death per 1.51 million episodes of exertion. Assuming a sexually active adult life of 50 years and a lifetime average frequency of sexual intercourse of once a week, only one out of 580 men might be expected to suffer a sudden death attributable to sexual intercourse.

Our study of sexual intercourse reported at baseline cannot explore whether there is any association between sexual activity and triggering of an acute stroke. The occurrence of acute stroke immediately after sexual intercourse has been reported by previous case studies, and these tend to be attributable to vasospasm, ${ }^{12}{ }^{13}$ cerebral haemorrhage or subarachnoid haemorrhage. ${ }^{1}$ In cerebral haemorrhage and subarachnoid haemorrhage, acute rises in blood pressure are of importance in triggering an event. It has been shown that male sexual intercourse induces transient increases in heart rate, blood pressure and plasma noradrenaline levels, ${ }^{14}$ and such rises might precipitate a haemorrhagic stroke in susceptible men. Single photon emission computed tomography studies have shown a decrease of cerebral blood flow during sexual intercourse in all cortical areas except in the right prefrontal cortex, where the cerebral blood flow increased..$^{15}$ Such changes would be unlikely to precipitate ischaemic stroke.

Headaches occurring during sexual activity are well recognised, ${ }^{16}{ }^{17}$ and it is possible that their occurrence would result in reduced frequency of sexual intercourse and any association with stroke would be masked. However, sexual headaches seem to be a relatively rare and benign complaint ${ }^{18}$ and would be unlikely to have a major effect on our findings. Use of antihypertensive drugs is associated with an increased risk of impotence ${ }^{19-21}$ and men receiving such treatment might be expected to report lower levels of sexual activity and an increased risk of stroke attributable to hypertension. The trends observed do not suggest that this mechanism is of importance but our data are limited.

It is possible that response bias has affected our findings. Not all men wanted to respond to the question about sexual intercourse, and they differed in systematic ways from those who did answer the question: they were older, shorter, had more coronary heart disease at baseline, and were more likely to be in manual occupations. It is likely that they had less active sex lives and it is also possible that they were at greater risk of stroke because of these other risk factors. Our finding that men reporting more sexual intercourse do not suffer an excess of strokes is unlikely to be affected by this response bias. It is possible that ascertainment of clinical events might be incomplete but it is unlikely that this would be biased by frequency of sexual intercourse and affect our findings.

It might be expected that a protective inverse relation between sexual intercourse and ischaemic stroke would be observed, as frequency of sexual intercourse might act as a proxy measure of physical activity that has been shown to protect against ischaemic stroke in both men ${ }^{22}$ and women. ${ }^{23}$ Perhaps the energy expenditure associated with sexual activity in middle aged men is insufficient to provide the protective effect seen with moderate to vigorous levels of other forms of physical activity. It is curious that frequency of sexual intercourse seems to protect against fatal coronary heart disease but not against ischaemic stroke. Updating of the CHD 


\section{Key points}

- Sexual intercourse may trigger the acute onset of coronary heart disease, sudden death and haemorrhagic stoke.

- Little scientific evidence exists on the role of sexual intercourse as a risk factor for coronary heart disease and stroke.

- Frequent sexual intercourse is associated with lower risk of fatal coronary heart disease, but this attenuates with duration of follow up.

- Frequency of sexual intercourse is not strongly associated with risk of ischaemic stroke.

mortality at 10 years of follow up reported previously, ${ }^{6}$ has attenuated the strong relation observed, which is probably because of the longer duration of follow up and the diminishing predictive value of baseline assessment of frequency of sexual intercourse.

While it has been suggested that the mortality findings are likely to be attributable to confounding rather than representing a true causal effect, ${ }^{624}$ the specificity of the relation with coronary heart disease and not stroke, and the failure to find a similar association with stroke mortality suggests that confounding may be an incomplete explanation of the coronary heart disease mortality findings. Both coronary heart disease and stroke incidence are positively correlated with decreased levels of physical activity, ${ }^{25}$ so if sexual intercourse were acting simply as a proxy for overall levels of physical activity, a similar relation would be expected with both diseases. However, stroke and coronary heart disease epidemiology differ in many ways and confounding of one exposure/disease association and not another is possible. Furthermore, the small number of events makes the shape of the exposure-disease association relatively uncertain. Sexual intercourse has been implicated in triggering both sudden death and non-fatal myocardial infarction, ${ }^{26}$ but the risks of both events are extremely low. Further exploration of the mechanisms by which differential effects of sexual intercourse on coronary heart disease and stroke risk arise might include effects on mood and personality and the role of male sex hormones.

Overall, middle aged men should be heartened to know that sexual intercourse is not likely to result in strokes, and that some protection from fatal coronary events may be an added bonus. Efforts to promote life prolonging and enhancing activity are central to much public health promotion. But without a clear understanding of the lay epidemiology of common, chronic diseases, rejection of health promotion activities is all too common by those with potentially most to gain. ${ }^{27}{ }^{28}$ Scientific epidemiology seldom derives its hypotheses from lay understanding of disease and consequently makes a much weaker contribution to public health programmes than might otherwise be the case.

\section{ACKNOWLEDGEMENTS}

We thank the participants, and the fieldworkers who collected data and specimens during phases I to IV of the Caerphilly study.

Funding: the Caerphilly Study is supported by grants from the MRC, Stroke Association and NHS RED programme.

Conflicts of interest: none.

\section{Authors' affiliations}

S Ebrahim, M May, Y Ben Shlomo, P McCarron, S Frankel, G Davey Smith, Department of Social Medicine, University of Bristol, UK

J Yarnell, Department of Epidemiology and Public Health, Queens

University of Belfast, Northern Ireland

\section{REFERENCES}

1 Jimbo H, Doi H, Matsumoto K, et al. Cerebrovascular diseases caused by sexual intercourse. Stroke: Abstracts from $4^{\text {th }}$ World Stroke Conference. Melbourne, November 2000:24

2 Ellis R. Sex is good for the heart. Daily Express 30 November 2000

3 Study links more sex, longer life. The Associated Press. Friday, December 19, 1997 http://amarillonet.com/stories/121997/ a.study.shtml

4 Gur AY, Bornstein NM. Are there any unique epidemiological and vascular risk factors for ischaemic strokes that occur in the morning hours?. Eur J Neurol 2000;7:179-81.

5 Bornstein NM, Gur AY, Fainshtein P, et al. Stroke during sleep epidemiological and clinical features. Cerebrovasc Dis 1999;9:320-2.

6 Davey Smith G, Frankel S, Yarnell J. Sex and death: are they related? Findings from the Caerphilly cohort study. BMV 1997:315:1641-5.

7 Caerphilly and Speedwell collaborative heart disease studies. The Caerphilly and Speedwell Collaborative Group. J Epidemiol Community Health 1984;38:259-62.

8 Greenwood R, McCarron P, Davey Smith G, et al. The Caerphilly and Speedwell Stroke study: I. Methods and incident events. Public Health 2001:115:4-11.

9 Davidson JM, Chen JJ, Crapo L, et al. Hormonal changes and sexual function in aging men. J Clin Endocrinol Metab 1983;57:71-7.

10 Strachan DP, Mendall MA, Carrington D, et al. Relation of Helicobacter pylori infection to 13-year mortality and incident ischaemic heart disease in the Caerphilly prospective heart disease study. Circulation 1998;98:1286-90.

11 Albert CM, Mittleman MA, Chae CU, et al. Triggering of sudden death from cardiac causes by vigorous exertion. N Engl J Med 2000;343:1355-61.

12 Levy RL. Stroke and orgasmic cephalgia. Headache 1981; 21:12-13.

13 Jackson $M$, Lennox $G$, Jaspan $T$, et al. Migraine angiitis precipitated by sex headache and leading to watershed infarction. Cephalalgia 1993; 13:427-30

14 Kruger T, Exton MS, Pawlak C, et al. Neuroendocrine and cardiovascular response to sexual arousal and sexual intercourse in men. Psychoneuroendocrinology 1998;23:401-11.

15 Tiihonen J, Kuikka J, Kupila J, et al. Increase in cerebral blood flow of right prefrontal cortex in man during sexual intercourse. Neurosci lett 1994; 170:241-3.

16 Lance JW. Headaches related to sexual activity. J Neurol Neurosurg Psychiatry 1976;39:1226-30.

17 Pascual J, Iglesias F, Oterino A, et al. Cough, exertional, and sexual headaches: an analysis of 72 benign and symptomatic cases. Neurology 1996;46:1520-4

18 Ostergaard JR, Kraft M. Benign coital headache. Cephalalgia $1992 ; 12: 353-5$

19 Cushman WC, Cohen JD, Jones RP, et al. Comparison of the fixed combination of enalapril/diltiazem ER and their monotherapies in stage 1 to 3 essential hypertension. Am J Hypertens 1998;11:23-30.

20 Ramsay LE, Yeo WW, Jackson PR. Thiazide diuretics: first-line therapy for hypertension. J Hypertens 1992;10:S29-32

21 Bulpitt CJ, Beevers DG, Butler A, et al. The effects of anti-hypertensive drugs on sexual function in men and women: a report from the DHSS Hypertension Care Computing Project (DHCCP). J Hum Hypertens 1989;3:53-6.

22 Wannamethee SG, Shaper AG. Physical activity and stroke in British middle-aged men. BMU 1992;304:597-601.

23 Hu FB, Stampfer M, Colditz GA, et al. Physical activity and risk of stroke in women. JAMA 2000;283:2961-7.

24 Hotopf $\mathbf{M}$, Wessely $\mathrm{S}$. The earth may move, but lets keep our feet on the ground. BM 1997;315:1645

25 Wannamethee SG, Shaper AG. Physical activity in the prevention of cardiovascular disease. An epidemiological perspective. Sports Med 2001;31:101-14.

26 Muller JE, Mittleman A, Maclure M, et al. Triggering myocardial infarction by sexual activity. Low absolute risk and prevention by regular physical exertion. JAMA 1996:275:1405-9.

27 Frankel S, Davison C, Davey Smith G. Lay epidemiology and the rationality of responses to health education. Br J Gen Pract $1991 ; 41: 428-30$

28 Davison C, Frankel S, Davey Smith G. The limits of lifestyle: re-assessing 'fatalism' in the popular culture of illness prevention. Soc Sci Med 1992;34:675-85. 